\title{
Boîtes en peau non tannée
}

\section{B. Dudot}

\section{OpenEdition}

\section{Journals}

Édition électronique

URL : http://journals.openedition.org/encyclopedieberbere/1783

ISSN : 2262-7197

\section{Éditeur}

Peeters Publishers

Édition imprimée

Date de publication : 1 décembre 1991

Pagination : 1559-1563

ISBN : 2-85744-549-0

ISSN : 1015-7344

\section{Référence électronique}

B. Dudot, « Boîtes en peau non tannée », Encyclopédie berbère [En ligne], 10 | 1991, document B87, mis en ligne le 01 mai 2013, consulté le 24 septembre 2020. URL : http://journals.openedition.org/ encyclopedieberbere/1783

Ce document a été généré automatiquement le 24 septembre 2020.

(c) Tous droits réservés 


\section{Boîtes en peau non tannée}

\section{B. Dudot}

1 L'artisanat d'Agadès compte une production très spécifique, celle des boîtes en peau épilée et parcheminée qu'on désigne du nom général de batta (au pluriel battochi) en haoussa, et de albattan en tamachek de l'Aïr.

2 Tombouctou, au Mali, en produit également.

3 Le $\mathrm{D}^{\mathrm{r}}$ Henri Barth, explorateur allemand au service du gouvernement anglais, premier européen à avoir traversé l'Aïr et séjourné à Agadès au début de la seconde moitié du XIXe siècle, vit ces boîtes sur le marché du quartier Katanga à Agadès et fut frappé par la beauté de leurs formes et l'originalité de leur décoration. Fr. de Zeltner, dans le Journal de la Société des Africanistes de 1932, a étudié quelques modèles de boîtes rapportées par elle de l'Aïr et de Tombouctou. En décembre 1968, j'ai pu étudier, avec l'écrivain suisse René Gardi, la technique de leur fabrication. Six familles d'Agadès fabriquaient la totalité des albattan dont la plus grande partie, de qualité médiocre, est achetée par les commerçants mozabites pour être revendue dans les oasis du Sud algérien. Les fabricants - maï batta ou maï battochi - avaient un chef de corporation, Maître Andillo, vieillard septuagénaire et sans conteste le plus habile de tous; ces artisans habitaient le quartier Obitara, au sud-est de la ville.

\section{Préparation de la peau}

On a très souvent dit que les boîtes étaient faites avec des mamelles de chamelles : il n'en est rien. La matière première est la peau de bœuf, de vache ou de chameau (les peaux de mouton et de chèvres sont trop minces). Toutes les parties de la peau peuvent être utilisées. La peau brute et sèche est ramollie dans l'eau pendant plusieurs jours, puis débarrassée par grattage des adhérences de chair et de graisse, enfin épilée et remise à tremper dans l'eau. 


\section{Confection du moule en terre}

5 L'artisan pétrit de l'argile débarrassée de tout caillou et en façonne des boules auxquelles il donne ensuite la forme voulue : tenant la boule de la main gauche, il tapote la forme à petits coups et sur toutes ses faces avec une espèce de palette taillée dans une planchette de bois blanc et portant à une extrémité un évidement légèrement concave; il existe plusieurs palettes de taille variable selon la grosseur du moule. Ce dernier est dénommé laka tandae en haoussa (de laka, terre argileuse, et tandae, pluriel de tandou désignant les boîtes rondes en peau).

6 Pour faciliter le démoulage ultérieur de la peau, qui se fait en cassant le moule, celui-ci est évidé partiellement quand la terre est encore malléable : l'artisan commence par découper avec une lame tranchante le fond légèrement aplati du moule en une épaisse rondelle qu'il met de côté ; puis il enlève la terre du centre du moule en laissant à la paroi 1 à $3 \mathrm{~cm}$ d'épaisseur, celle-ci variant avec la grosseur du modèle désiré. Puis le fond est remis à sa place et recollé avec un peu de glaise. Le moule va sécher à l'ombre un jour entier.

\section{Modelage de la peau sur le moule}

7 L'artisan commence par frotter la surface du moule avec une terre calcaire blanche réduite en poudre : cet enduit empêchera la peau mouillée de coller à l'argile et facilitera le démoulage. Ayant essoré la peau, il l'étale sur une large pierre plate et lisse et la gratte pour en enlever les derniers vestiges de poils, de chair ou de graisse avec une sorte de tranchet de forme spéciale, composé d'une lame de fer trapézoïdale à tranchant courbe et d'un manche de bois court. L'outil est tenu manche appuyé sur la paume de la main et maintenu par trois doigts, l'index et le majeur appuyés sur la lame tenue presque à l'horizontale. Le modelage de la peau se fait en deux étapes : la boîte d'abord, le couvercle ensuite.

Partie A. On découpe dans la peau une bande de 10 à $12 \mathrm{~cm}$ de largeur et on l'applique sur les côtés du moule, laissant à découvert la partie supérieure conique et la partie centrale au fond ; la peau est lissée soigneusement pour éviter les bulles d'air et les faux plis; le fond est recouvert de déchets de peau et sur le tout est appliquée une pièce de peau arrondie aux bords très amincis de sorte que le raccord entre le fond et la peau des côtés est absolument invisible après séchage; d'ailleurs, quelle que soit la partie modelée, la peau est toujours très finement amincie pour obtenir des raccords imperceptibles.

9 Partie B. Avant d'appliquer sur la partie A la peau formant couvercle, la pointe conique est enduite de calcaire en poudre. Les bords de la partie A sont égalisés au tranchet, puis enduits d'un peu de graisse pour éviter l'adhérence à la peau du couvercle qui va les recouvrir sur 2 à $3 \mathrm{~cm}$. La peau $B$ est appliquée sur la pointe et bien étirée en tous sens pour épouser parfaitement la forme du moule. Les bords de B sont ensuite liés avec une lanière en feuille de doum pour éviter le retrait de la peau au séchage qui dure un jour à l'ombre ; quand la peau est encore un peu souple, les bords en sont taillés audessous de la ligature. 
10 Pour le modelage des boîtes de petite taille, la partie A est faite d'une seule pièce de peau. Pour les boîtes de très petite taille, le moule d'argile n'est pas évidé car il peut se briser facilement après séchage.

\section{Décoration et teinture des boîtes}

11 Cette partie du travail est réservée aux femmes des artisans. La décoration porte toujours sur les couvercles des boîtes, l'autre partie étant simplement teinte. Le tracé du décor sur les couvercles est obtenu par l'application de fils de cire roulés à la main selon des dessins géométriques très simples : lignes parallèles, en chevrons, en zigzag, triangles, spirales, cercles, points, losanges, croix... Puis la boîte est placée dans une bassine où elle est arrosée de teinture ; après séchage, les fils de cire sont enlevés et les dessins décoratifs apparaissent en clair (de la teinte de la peau parcheminée) sur un fond rouge-brun. Il convient de faire remarquer que ces opérations se font avant le démoulage, car la boîte vide ne supporterait plus d'être humidifiée.

12 Voici les détails des procédés :

- La femme tient sur ses genoux une planchette sur laquelle elle confectionne les fils de cire : elle fait rouler une boulette de cire d'abeille entre la surface légèrement convexe d'une rondelle découpée dans une calebasse et la surface plane de la planchette ; la boulette devient un petit boudin qui, en s'étirant et s'amincissant de plus en plus, se transforme en un fil régulier de longueur variable atteignant $30 \mathrm{~cm}$ et plus.

- Les fils de cire étant prêts, l'opératrice tient la boîte appuyée sur ses jambes croisées. La main gauche tient le fil et le guide, tandis que la main droite l'applique sur le couvercle en le faisant adhérer d'une légère pression des doigts sans cependant l'écraser : c'est là que réside l'habileté de la femme ; on peut remarquer en effet que les plus belles boîtes sont celles où les parties claires sont les plus étroites, donc où les fils étaient appliqués juste ce qu'il fallait pour tenir sur la peau.

Le fil trop long est coupé à l'aide de l'ongle; le pouce joue le rôle le plus actif dans l'application du fil.

14 Ce travail se pratique à l'air libre, par un jour ensoleillé, la chaleur de l'air ambiant favorisant l'adhérence de la cire sur la boîte. La disposition harmonieuse des motifs décoratifs sur les boîtes dépend uniquement de l'appréciation visuelle, puis de l'habileté et du doigté de l'opératrice.

- Tous les fils étant appliqués, la boîte est posée dans un vieux canari (pot de terre cuite à large ouverture) dont le fond est rempli de teinture rouge (karandafi en haoussa) obtenue en faisant macérer dans de l'eau, avec un peu de natron, des feuilles de mil séchées et pilées. La boîte est arrosée de ce liquide de telle sorte que toutes les parties non couvertes de cire soient imprégnées; puis la boîte est retirée et mise à sécher une demi-heure; ensuite la cire est enlevée avec un chiffon, laissant apparaître la décoration en clair. 


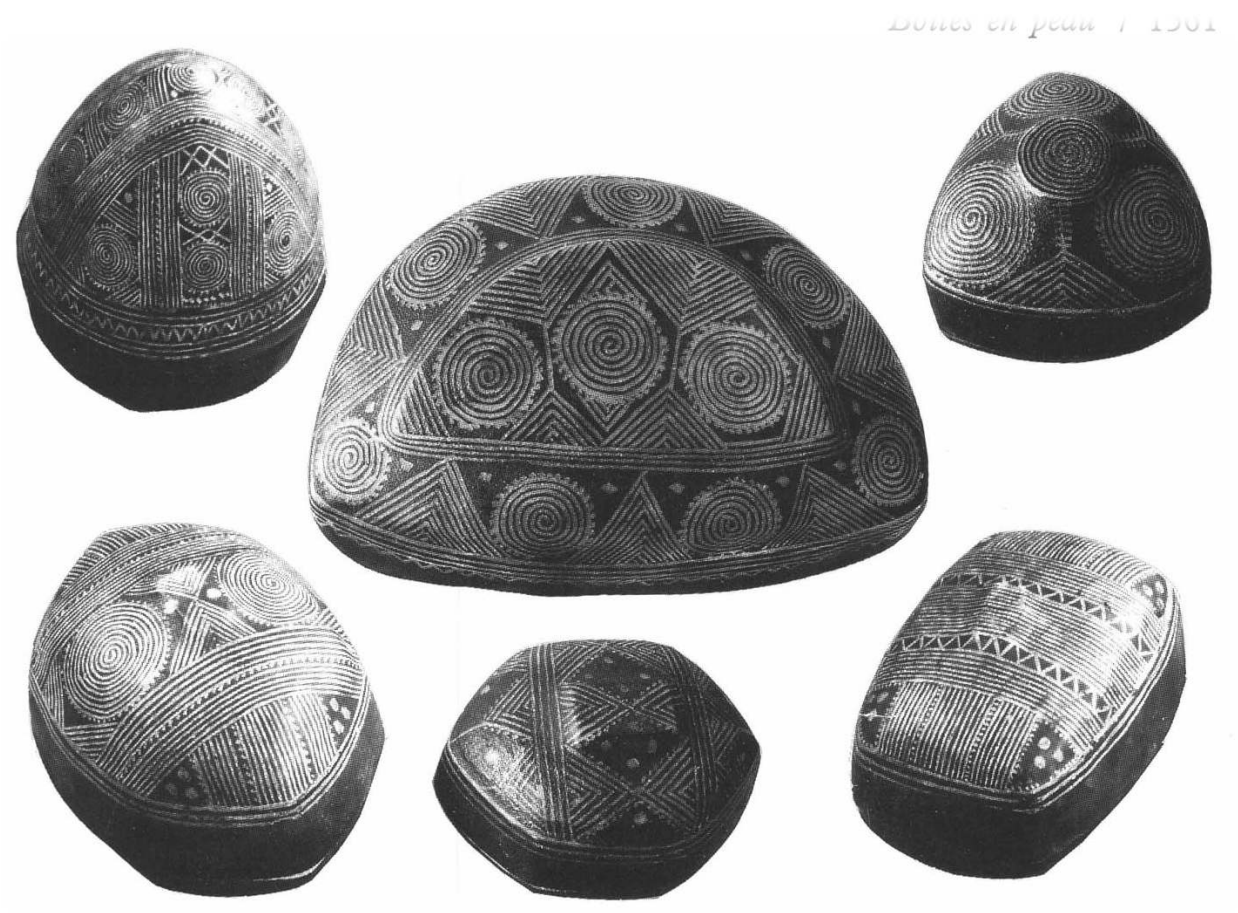

\section{Démoulage des boîtes}

L'artisan tient la boîte dans la main gauche et en frappe le pourtour à petits coups secs avec un caillou bien poli. Le moule évidé ne tarde pas à se fendre et à se briser à l'intérieur de la boîte. Le couvercle se détache et la terre est enlevée. Pour démouler les boîtes de petite taille dont le moule n'est pas évidé, on frappe celles-ci contre la grande pierre plate servant au nettoyage des peaux ; la terre finit par se fractionner puis, après enlèvement du couvercle, la boîte est nettoyée.

\section{Récipients en peau non tannée, à usages ménagers}

Le maitre Andillo confectionnait également des récipients destinés à contenir ou transporter des matières liquides ou pâteuses : huile, beurre, graisse ou miel; à l'exception de tout liquide aqueux qui rendrait le récipient inutilisable en le ramollissant. Ces récipients ont un corps sphérique de 10 à $12 \mathrm{~cm}$ de diamètre, surmonté d'un large col haut d'une douzaine de $\mathrm{cm}$, coiffé d'un couvercle à peu près hémisphérique. Ils ont l'aspect d'une grosse gourde, d'une contenance de 2 à 3 litres. Deux petites anses de peau fixées de part et d'autre du corps, et une troisième anse au sommet du couvercle permettent, tout à la fois, de porter et suspendre le vase et de maintenir le couvercle fermé grâce à une lanière de cuir reliant les trois anses.

Andillo nommait ce récipient : tazefeness; la plupart des gens d'Agadès le nomment : tandou (en haoussa) et les Touaregs de l'Aïr : etchen wiji (littéralement : récipient à huile).

18 Leur fabrication est assez semblable à celle des batta. Il convient cependant de noter les différences suivantes : 
- Le moule de terre est fait d'une boule évidée surmontée d'un cylindre légèrement conique et arrondi au sommet, également évidé ; la boule et le cylindre sont fixés l'un sur l'autre par un joint de glaise.

- La peau mouillée est modelée sur la sphère d'abord, puis sur le col ; ensuite sont fixées les deux petites anses. Après séchage, le couvercle est façonné avec son anse. Le fond et les côtés de la sphère sont recouverts d'une pièce de peau non épilée qui renforce sa solidité et son étanchéité et lui sert aussi de décoration, car ce récipient n'est ni décoré à la cire ni teint comme les autres boîtes de peau.

19 Autrefois, les fabricants de boîtes en peau façonnaient avec la même matière de petits animaux, chevaux et chameaux, pour servir de jouets aux enfants.

\section{Différents modèles de boîtes} l'Air).

- Toulon : Batta dont le sommet du couvercle est aplati ; sa forme rappelle la jarre à eau, (toulou, en haoussa).

- Tandou : boîte sphérique de différentes tailles, utilisée très souvent pour garder la gomme odorante ou l'encens (touraré, en haoussa) brûlée dans les maisons; le nom tamachek de cette boîte est albadeni.

- Sandouki : boîte de forme rectangulaire (sandouki, en haoussa signifie caisse).

- Kounkourou : boîtes en forme d'amande ou ovales, losangiques, polygonales (kounkourou en haoussa signifie tortue).

- Tasambow : désigne les boîtes triangulaires, par analogie de forme avec les plaques de sel gemme de la saline de Teguidda-n-Tesemt, portant ce nom.

- Wata : boîte en forme de demi-lune; Tellit en tamache (Wata et Tellit désignent la lune).

- Feifei : boîtes rondes et aplaties (Feifei, en haoussa, désigne le van de paille tressée).

- Koutoumbi : ce sont des boîtes dont le couvercle porte une boîte plus petite à son sommet. Il existe deux variantes :

a. boîte agrémentée de deux ou quatre petites anses sur les côtés et de cinq autres groupées sur le couvercle de la boîte sommitale.

b. boîte portant sur le couvercle deux, trois ou quatre petites boîtes disposées symétriquement.

- Madembachi : double boîte faite de deux batta superposées et réunies par un large col. Elle rappelle la forme d'un couscoussier, d'où son nom : madembachi ou assedembou en tamachek de l'Aïr (litt. qui fait gonfler la farine).

- Kambou fitila : ressemble à la précédente, mais avec un col plus étroit entre les deux boîtes : la fragilité qui en découle est compensée par trois ou quatre colonnettes de soutien en peau, disposées symétriquement.

21 L'ensemble a l'apparence de certaines lampes à pression à gaz de pétrole, en usage en Afrique durant de nombreuses années, surtout en brousse (Kambou fitila signifie littéralement : ceinture de la lampe).

- Gorouba : se compose de trois tandou superposées ; gorouba désigne les noix du palmier doum, groupées sur une tige commune. Se nomme tamergit en tamachek de l'Aïr. 


\section{BIBLIOGRAPHIE}

Zeltner F. de, « Les boîtes en cuir du Soudan », Journal de la Soc. des Africanistes, t. 2, 1932, pp.

23-24.

Lhote H., « Les boîtes moulées du Soudan dites "bata" », Bull, de l'I.F.A.N., t. 14, 1952, pp. 919-955.

Dudot B., « Une production originale de l'artisanat d'Agadez : les boîtes en peau non tannée dites "Batta" ", Notes africaines, n 122, 1969, pp. 55-58.

INDEX

Mots-clés : Ethnographie, Sahara 